\title{
Article \\ Genetic Risk and Phenotype Correlation of Primary Open-Angle Glaucoma Based on Rho-Kinase Gene Polymorphisms
}

\author{
Yong-Woo Kim (D), Eunoo Bak, Seoyoung Wy, Seung-Chan Lee, Yu-Jeong Kim, Young-Kook Kim, Ki-Ho Park \\ and Jin-Wook Jeoung *(i)
}

check for

updates

Citation: Kim, Y.-W.; Bak, E.; Wy, S.; Lee, S.-C.; Kim, Y.-J.; Kim, Y.-K.; Park, K.-H.; Jeoung, J.-W. Genetic Risk and Phenotype Correlation of Primary Open-Angle Glaucoma Based on Rho-Kinase Gene Polymorphisms. J. Clin. Med. 2021, 10, 1953. https:// doi.org/10.3390/jcm10091953

Academic Editor:

Andrzej Grzybowski

Received: 4 April 2021

Accepted: 27 April 2021

Published: 1 May 2021

Publisher's Note: MDPI stays neutral with regard to jurisdictional claims in published maps and institutional affiliations.

Copyright: (c) 2021 by the authors. Licensee MDPI, Basel, Switzerland. This article is an open access article distributed under the terms and conditions of the Creative Commons Attribution (CC BY) license (https:/ / creativecommons.org/licenses/by/ $4.0 /)$.
Department of Ophthalmology, Seoul National University Hospital, Seoul 03080, Korea; yongwookim@snu.ac.kr (Y.-W.K.); eunoo629@gmail.com (E.B.); wsy445@gmail.com (S.W.); tmdcks87@naver.com (S.-C.L.); yjkimhappy@hanmail.net (Y.-J.K.); md092@naver.com (Y.-K.K.); kihopark@snu.ac.kr (K.-H.P.)

* Correspondence: neuroprotect@gmail.com; Tel.: +82-2-2072-2438; Fax: +82-2-741-3187
Abstract: Rho-associated coiled-coil kinase (ROCK) signaling can affect glaucoma risk by regulating trabecular meshwork outflow. We investigated the effect of ROCK gene polymorphism on the risks of primary open-angle glaucoma (POAG) and POAG-related phenotypes including intraocular pressure (IOP) in a Korean population. A total of 24 single-nucleotide polymorphisms (SNPs) from ROCK1 and ROCK2 were selected and genotyped for 363 POAG patients and 213 healthy controls. Among the 363 POAG patients, 282 were normal-tension glaucoma (NTG, baseline IOP $\leq 21 \mathrm{mmHg}$ ) and 81 were high-tension glaucoma (HTG, baseline IOP > $21 \mathrm{mmHg}$ ). The SNPs rs288979, rs1006881, rs35996865, rs10083915, and rs11873284 in ROCK1 (tagged to each other, $r^{2}=1$ ) were nominally associated with risk of HTG $(\mathrm{OR}=0.52, p=0.045)$. However, there were no SNPs that were significantly associated with the risk of NTG. In the genotype-phenotype correlation analysis, the SNPs rs2230773 and rs3771106 in ROCK2 were significantly correlated with central corneal thickness (CCT)-adjusted IOP $(p=0.024)$ and axial length (AXL; $p=0.024)$, respectively. The present data implicated the role of ROCK in POAG development, and as such, can serve as a good reference for upcoming Rho/ROCK-pathway-related studies on POAG.

Keywords: rho-associated coiled-coil kinase (ROCK); single nucleotide polymorphism; primary open-angle glaucoma

\section{Introduction}

Rho-associated coiled-coil kinase $(R O C K)$ is a member of the serine/threonine protein kinase family that serves as a major downstream effector of the Rho pathway [1,2]. ROCK attaches to Rho and forms a Rho/ROCK complex that regulates actin-myosin dynamics and is involved in multiple physiological functions, such as cell contraction, migration, proliferation, angiogenesis, chemotaxis, neural protection, and vasodilatation [3,4]. In humans, ROCK has two isoforms, ROCK1 and ROCK2, each of which is separately encoded on chromosome 18 (18q11.1) and chromosome 2 (2p24), respectively [5].

ROCK molecules are ubiquitous in all cellular tissues and organs, including ocular tissues such as the cornea, trabecular meshwork (TM), iris, and retina [6]. Recently, ROCK has drawn increased clinical interest in the field of glaucoma due to the improved understanding of its role in intraocular pressure (IOP) regulation [7]. ROCK inhibitors can lower IOP by modifying the cytoskeleton in Schlemm's canal and relaxing smooth muscle cells in the TM. It is well documented that TM cells express both ROCK1 and ROCK2 as well as downstream effectors of the ROCK pathway, including myosin light chain (MLC), Lin11/Isl-1/Mec-3 (LIM) kinase, and cofilin [8]. These findings support the hypothesis that ROCK inhibitors can lower IOP by reducing outflow resistance. ROCK inhibitors are also 
known for their neuroprotective effect on retinal ganglion cells (RGC) via improved ocular blood flow, RGC survival, and axonal regeneration $[9,10]$. Therefore, ROCK inhibitors currently are under investigation as potential therapeutic targets for primary open-angle glaucoma (POAG).

In this regard, the ROCK gene polymorphisms may play a role in stratifying the risk of POAG on a genetic basis. The purpose of the present study, accordingly, was to investigate possible associations between ROCK gene variants and POAG development in a Korean population. In addition, the genotype-phenotype correlation between ROCK gene variants and clinical features of POAG, including IOP, were explored.

\section{Methods}

The present study was undertaken as a part of the GLAU-GENDISK (GLAUcoma GENe DIscovery Study in Korea) project, which is an ongoing prospective study designed and inaugurated in 2011 [11]. The primary objective of the GLAU-GENDISK project is to investigate and identify novel genetic variants for various types of glaucoma in a Korean population. The secondary objectives include the establishment of the genotype-phenotype relationships in glaucoma patients and the construction of new disease prediction models. This study was approved by the Seoul National University Hospital Institutional Review Board and followed the tenets of the Declaration of Helsinki (1964). Written informed consent was obtained from each of the enrolled subjects.

\subsection{Study Subjects}

All of the subjects included in this analysis were Korean. They included 363 patients with POAG and 213 healthy controls, all 576 of whom had been enrolled in the GLAUGENDISK. POAG was defined as the presence of glaucomatous optic disc changes with corresponding glaucomatous visual field (VF) defects and an open-angle confirmed by gonioscopy. Glaucomatous optic disc changes were defined as neuroretinal rim thinning, notching, excavation, or retinal nerve fiber layer (RNFL) defects. Glaucomatous VF defects were defined as (1) glaucoma hemifield test values outside the normal limits, (2) three or more abnormal points with a probability of being normal of $p<0.05$, of which at least one point has a pattern deviation of $p<0.01$, or (3) a pattern standard deviation of $p<0.05$. The VF defects were confirmed on two consecutive reliable tests (fixation loss rate $\leq 20 \%$, false-positive and false-negative error rates $\leq 25 \%$ ). The baseline IOP value was defined as the mean of at least two measurements before initiation of IOP-lowering treatment. Based on the baseline IOP values, high-tension glaucoma (HTG) eyes were defined as POAG eyes with a baseline IOP of greater than $21 \mathrm{~mm} \mathrm{Hg}$, and normal-tension glaucoma (NTG) eyes were defined as POAG eyes with a baseline IOP of less than or equal to $21 \mathrm{~mm} \mathrm{Hg}$.

The present study excluded subjects with (1) a history of retinal diseases such as age-related macular degeneration, epiretinal membrane, or diabetic retinopathy; or (2) insufficient measurement of baseline IOP.

The POAG patients in the GLAU-GENDISK cohort underwent a complete ophthalmic examination, including a visual acuity assessment, slit-lamp biomicroscopy, gonioscopy, Goldmann applanation tonometry, refractions, dilated fundus examination, disc stereophotography, red-free fundus photography using a digital fundus camera (VISUCAM, Carl Zeiss Meditec, Inc., Dublin, CA, USA), and standard automated perimetry (Humphrey C 24-2 SITA-Standard visual field; Carl Zeiss Meditec, Inc.). The CCT (Pocket II; Quantel Medical, Clermont-Ferrand, France) and AXL (AXIS-II Ultrasonic Biometer; Quantel Medical S.A., Bozeman, MT, USA) were measured as well. A $200 \times 200$ optic disc cube scan and a $200 \times 200$ macular cube scan were performed using Cirrus HD-OCT (Carl-Zeiss Meditec, Inc., Dublin, CA, USA), and the average peripapillary RNFL and macular ganglion cell-inner plexiform layer (GCIPL) thicknesses were measured with the built-in analysis algorithm (software version 6.0; Carl Zeiss Meditec, Inc., Dublin, CA, USA). For the POAG patients, the eye with the worse visual field mean deviation (VF MD) was selected for the 
analysis. For the healthy control, one eye was randomly selected using the sample function in R ( $\mathrm{R}$ version 3.6.1., available at: http:/ / www.r-project.org; accessed on 20 March 2020).

\subsection{Target SNP and Genotyping}

Candidate single-nucleotide polymorphisms (SNPs) of ROCK1 and ROCK2 were selected from reported SNPs that had been previously investigated for association with various diseases in other ethnicities [12]. A total of 24 SNPs (12 from ROCK1 and another 12 from ROCK2) were selected and genotyped (Table 1). Genotyping reactions were performed using the BioMark HD system (Fluidigm 192.24 SNPtypeTM, San Francisco, CA, USA). The primer pools were designed for specific target amplifications as well as allele-specific and locus-specific primers for detection of candidate SNPs (Table S1). The additional workflow was conducted according to the manufacturer's instructions for use of the Integrated IFC Controller RX, FC1 Cycler, and EP1 Reader (Fluidigm Corp., San Francisco, CA, USA). Signal intensities for genotype calling were scanned using the EP1 data collection and SNP Genotyping analysis software (version 4, Fluidigm Corp., San Francisco, CA, USA).

Table 1. Target SNPs for ROCK polymorphism analysis

\begin{tabular}{|c|c|c|c|c|c|c|c|}
\hline $\mathbf{N}$ & ID & $\begin{array}{c}\text { Tagging SNP } \\
\left(r^{2}=1\right)\end{array}$ & Gene & Chr & Position & Location & $\begin{array}{c}\text { AA } \\
\text { Change }\end{array}$ \\
\hline 1 & rs2271621 & rs2290156 & ROCK2 & 2 & 11193868 & intron & \\
\hline 2 & rs34945852 & & ROCK2 & 2 & 11197557 & missense & K997M \\
\hline 3 & rs202027620 & & ROCK2 & 2 & 11200967 & missense & T881I \\
\hline 4 & rs35768389 & & ROCK2 & 2 & 11214974 & missense & D515V \\
\hline 5 & rs190769228 & & ROCK2 & 2 & 11215516 & missense & $\mathrm{N} 445 \mathrm{H}$ \\
\hline 6 & rs2230773 & & ROCK2 & 2 & 11249688 & synonymous & \\
\hline 7 & rs3771106 & rs2230774, rs1515219, rs726843, rs978906 & ROCK2 & 2 & 11249986 & intron & \\
\hline 8 & rs965665 & & ROCK2 & 2 & 11258749 & intron & \\
\hline 9 & rs10178332 & & ROCK2 & 2 & 11268891 & intron & \\
\hline 10 & rs1130157 & & ROCK2 & 2 & 11286615 & 5'UTR & \\
\hline 11 & rs6755196 & & ROCK2 & 2 & 11320199 & intron & \\
\hline 12 & rs10929732 & & ROCK2 & 2 & 11343366 & intron & \\
\hline 13 & rs75122528 & & ROCK1 & 18 & 20947821 & 3'UTR & \\
\hline 14 & rs2847081 & & ROCK1 & 18 & 20948500 & 3'UTR & \\
\hline 15 & rs8089184 & & ROCK1 & 18 & 20970650 & intron & \\
\hline 16 & rs288980 & & ROCK1 & 18 & 21029619 & intron & \\
\hline 17 & rs288979 & rs7239317 & ROCK1 & 18 & 21031282 & intron & \\
\hline 18 & rs2127958 & & ROCK1 & 18 & 21073649 & intron & \\
\hline 19 & rs1481280 & & ROCK1 & 18 & 21075490 & intron & \\
\hline 20 & rs1006881 & rs11874761 & ROCK1 & 18 & 21101332 & intron & \\
\hline 21 & rs35996865 & & ROCK1 & 18 & 21112383 & 5'near & \\
\hline 22 & rs10083915 & & ROCK1 & 18 & 21120994 & 5'near & \\
\hline 23 & rs11873284 & & ROCK1 & 18 & 21135030 & 5'near & \\
\hline 24 & rs1515210 & & ROCK1 & 18 & 29283684 & $5^{\prime}$ & \\
\hline
\end{tabular}

SNP: single nucleotide polymorphism, ROCK: rho-kinase, Chr: chromosome, AA: amino acid, UTR: untranslated region.

\subsection{Data Analysis}

The SNP genotype frequencies were examined for Hardy-Weinberg equilibrium based on the corresponding chi-squared statistics. Continuous variables were compared between the groups by Student's $t$-test. Data were analyzed using unconditional logistic regression, controlling for age and sex, in order to calculate the odds ratio (OR) as an estimate of the relative risk of POAG associated with the SNP genotype. The key POAG-related phenotypes derived from the 363 eyes of the 363 POAG patients, including IOP, history of disc hemorrhage, mean deviation (MD) of $\mathrm{VF}, \mathrm{AX}$, refraction, rim area, disc area, average cup-to-disc ratio (C/D), vertical C/D, cup volume, average RNFL thickness, average GCIPL thickness, and family history of glaucoma, were evaluated for SNP-genotype correlation 
after adjustment for age and sex (additive model). In the case of IOP, the additive model was built with adjustment for CCT as well as age and sex. For average RNFL and GCIPL thicknesses, signal strength was also considered for the additive model. Statistical analysis was conducted by $\mathrm{R}$ software ( $\mathrm{R}$ version 3.6.1., available at: http:/ /www.r-project.org; accessed 20 March 2020).

\section{Results}

The present study included 363 POAG patients and 213 healthy controls. There were no significant differences in age $(54.0 \pm 13.7$ vs. $54.6 \pm 9.7$ years, $p=0.55$, values are mean \pm standard deviation) or sex (female, $180(49.6 \%)$ vs. $93(43.7 \%), p=0.17)$ between the two groups. Among the 363 POAG patients, 282 were NTG and 81 were HTG. The baseline IOP was $15.3 \pm 3.0 \mathrm{~mm} \mathrm{Hg}$ and $26.0 \pm 6.5 \mathrm{~mm} \mathrm{Hg}$, respectively. The HTG eyes had significantly thicker CCT $(546.9 \pm 30.2 \mu \mathrm{M})$ than did the NTG eyes $(532.9 \pm 35.9 \mu \mathrm{m}$, $p<0.001)$. The HTG eyes also had thinner average RNFL $(64.3 \pm 14.3 \mu \mathrm{m})$ and GCIPL $(63.5 \pm 11.0 \mu \mathrm{M})$ thicknesses and lower MD of VF $(-14.5 \pm 10.0 \mathrm{~dB})($ all $p$-s $<0.05)$. The NTG eyes had a higher number of histories of disc hemorrhage (36 (12.8\%)) than did the HTG eyes $(3(3.7 \%), p=0.034)$. A Comparison of the ophthalmic demographics between NTG and HTG is provided in Table 2.

Table 2. Subject demographics

\begin{tabular}{|c|c|c|c|c|}
\hline Variable & \multicolumn{2}{|c|}{ POAG $(n=363)$} & Healthy $(n=213)$ & $p$-Value \\
\hline Age, year & \multicolumn{2}{|c|}{$54.0 \pm 13.7$} & $54.6 \pm 9.7$ & $0.55 *$ \\
\hline NTG & \multicolumn{2}{|c|}{$54.3 \pm 13.3$} & $54.6 \pm 9.7$ & $0.76^{*}$ \\
\hline HTG & \multicolumn{2}{|c|}{$53.1 \pm 15.3$} & $54.6 \pm 9.7$ & $0.40 *$ \\
\hline Sex (Female), $n(\%)$ & \multicolumn{2}{|c|}{$180(49.6 \%)$} & $93(43.7 \%)$ & $0.17+$ \\
\hline NTG & \multicolumn{2}{|c|}{$155(55.0 \%)$} & $93(43.7 \%)$ & $0.016+$ \\
\hline HTG & \multicolumn{2}{|c|}{$25(30.9 \%)$} & $93(43.7 \%)$ & $0.06+$ \\
\hline & NTG $(n=282)$ & HTG $(n=81)$ & & \\
\hline Baseline IOP, $\mathrm{mm} \mathrm{Hg}$ & $15.3 \pm 3.0$ & $26.0 \pm 6.5$ & & $<0.001 *$ \\
\hline $\mathrm{CCT}, \mu \mathrm{m}$ & $532.9 \pm 35.9$ & $546.9 \pm 30.2$ & & $0.001 *$ \\
\hline $\mathrm{AXL}, \mathrm{mm}$ & $24.7 \pm 1.6$ & $24.7 \pm 1.8$ & & 0.96 * \\
\hline DH history & $36(12.8 \%)$ & $3(3.7 \%)$ & & $0.034^{\dagger}$ \\
\hline Rim area, $\mathrm{mm}^{2}$ & $0.77 \pm 0.20$ & $0.67 \pm 0.29$ & & $0.013 *$ \\
\hline Disc area, $\mathrm{mm}^{2}$ & $1.95 \pm 0.50$ & $1.93 \pm 0.40$ & & $0.80 *$ \\
\hline Vertical C/D & $0.77 \pm 0.11$ & $0.81 \pm 0.11$ & & 0.024 * \\
\hline Average RNFL thickness, $\mu \mathrm{M}$ & $70.9 \pm 11.6$ & $64.3 \pm 14.3$ & & $0.001 *$ \\
\hline Average GCIPL thickness, $\mu \mathrm{M}$ & $67.9 \pm 8.8$ & $63.5 \pm 11.0$ & & 0.004 * \\
\hline MD of VF, $\mathrm{dB}$ & $-8.4 \pm 6.4$ & $-14.5 \pm 10.0$ & & $<0.001$ * \\
\hline
\end{tabular}

Data are presented as mean \pm standard deviation values. Statistically significant values are shown in bold. * Comparison performed using Student's $t$-test,${ }^{\dagger}$ Comparison performed using chi-square test. POAG: primary open-angle glaucoma, NTG: normal-tension glaucoma, HTG: high-tension glaucoma. IOP: intraocular pressure, CCT: central corneal thickness, AXL: axial length, DH: disc hemorrhage, C/D: cup-to-disc ratio, RNFL: retinal nerve fiber layer, GCIPL: ganglion cell-inner plexiform layer, MD: mean deviation, VF: visual field.

All of the SNPs were in Hardy-Weinberg equilibrium (all $p$-s $>0.05$ ). The SNPs rs34945852, rs35768389, rs190769228, and rs1130157 from ROCK2 were monomorphic in the present population, and therefore, minor alleles were not identified. The following SNPs were found to be in linkage disequilibrium (LD) $\left(r^{2}>0.95\right)$ : ROCK1, SNPs rs75122528, rs1481280, rs2847081, rs8089184, rs288979, rs1006881, rs35996865, rs10083915, and rs11873284; ROCK2, SNPs rs965665, rs10178332, and rs6755196.

In the association analysis, none of the SNPs from ROCK1/ROCK2 were significantly associated with risk of POAG (Table 3). In the subgroup analysis, the SNPs rs288979, rs1006881, rs35996865, rs10083915, and rs11873284 in ROCK1 (tagged to each other, $r^{2}=1$ ) were nominally associated with risk of HTG $(\mathrm{OR}=0.52, p=0.045)$. However, there were no SNPs that were significantly associated with risk of NTG (Table 4). 
Table 3. Genetic association of ROCK polymorphism with POAG

\begin{tabular}{|c|c|c|c|c|c|c|}
\hline \multirow{2}{*}{ Gene } & \multirow{2}{*}{ ID } & \multirow{2}{*}{ Alleles } & \multicolumn{4}{|c|}{ POAG } \\
\hline & & & Case MAF & Control MAF & OR $(95 \% \mathrm{CI})$ & $p$-Value \\
\hline ROCK2 & rs2271621 & $\mathrm{G}>\mathrm{T}$ & 0.486 & 0.479 & $1.03(0.81,1.32)$ & 0.80 \\
\hline ROCK2 & rs202027620 & $G>A$ & 0.000 & 0.005 & & \\
\hline ROCK2 & rs2230773 & $\mathrm{C}>\mathrm{T}$ & 0.024 & 0.024 & $1.04(0.47,2.33)$ & 0.92 \\
\hline ROCK2 & rs3771106 & $\mathrm{G}>\mathrm{A}$ & 0.448 & 0.433 & $1.06(0.83,1.36)$ & 0.63 \\
\hline ROCK2 & rs965665 & $G>C$ & 0.008 & 0.002 & $3.69(0.44,31.01)$ & 0.17 \\
\hline ROCK2 & rs10178332 & $A>C$ & 0.008 & 0.002 & $3.69(0.44,31.01)$ & 0.17 \\
\hline ROCK 2 & rs6755196 & $\mathrm{G}>\mathrm{A}$ & 0.008 & 0.002 & $3.68(0.44,30.94)$ & 0.17 \\
\hline ROCK2 & rs10929732 & $\mathrm{G}>\mathrm{A}$ & 0.036 & 0.045 & $0.80(0.44,1.47)$ & 0.48 \\
\hline ROCK1 & rs75122528 & $A>T$ & 0.300 & 0.319 & $0.92(0.71,1.19)$ & 0.54 \\
\hline ROCK1 & rs2847081 & $\mathrm{T}>\mathrm{C}$ & 0.138 & 0.127 & $1.09(0.76,1.56)$ & 0.65 \\
\hline ROCK1 & rs8089184 & $\mathrm{T}>\mathrm{C}$ & 0.145 & 0.131 & $1.11(0.78,1.58)$ & 0.56 \\
\hline ROCK1 & rs288980 & $\mathrm{T}>\mathrm{C}$ & 0.475 & 0.474 & $1.00(0.79,1.27)$ & 0.99 \\
\hline ROCK1 & rs288979 & $A>G$ & 0.138 & 0.129 & $1.08(0.76,1.54)$ & 0.67 \\
\hline ROCK1 & rs2127958 & $\mathrm{T}>\mathrm{C}$ & 0.441 & 0.445 & $0.99(0.78,1.25)$ & 0.90 \\
\hline ROCK1 & rs1481280 & $\mathrm{C}>\mathrm{A}$ & 0.300 & 0.312 & $0.95(0.74,1.23)$ & 0.72 \\
\hline ROCK1 & rs1006881 & $\mathrm{G}>\mathrm{A}$ & 0.140 & 0.129 & $1.10(0.77,1.56)$ & 0.61 \\
\hline ROCK1 & rs35996865 & $\mathrm{T}>\mathrm{G}$ & 0.138 & 0.129 & $1.08(0.76,1.54)$ & 0.68 \\
\hline ROCK1 & rs10083915 & $A>G$ & 0.141 & 0.129 & $1.10(0.77,1.56)$ & 0.60 \\
\hline ROCK1 & rs11873284 & $A>G$ & 0.141 & 0.129 & $1.10(0.77,1.57)$ & 0.60 \\
\hline ROCK1 & rs1515210 & $\mathrm{C}>\mathrm{G}$ & 0.183 & 0.181 & $1.03(0.75,1.41)$ & 0.86 \\
\hline
\end{tabular}

POAG: primary open-angle glaucoma, ROCK: rho-kinase, MAF: minor allele frequency, OR: odds ratio, CI: confidence interval. SNPs rs34945852, rs35768389, rs190769228, and rs1130157 in ROCK2 were monomorphic in cases and controls and were removed from the table.

Table 4. Genetic association of ROCK polymorphism with NTG and HTG

\begin{tabular}{|c|c|c|c|c|c|c|c|c|c|}
\hline \multirow{2}{*}{ Gene } & \multirow{2}{*}{ ID } & \multirow{2}{*}{ Alleles } & \multirow{2}{*}{$\begin{array}{c}\text { Control } \\
\text { MAF }\end{array}$} & \multicolumn{3}{|c|}{ NTG } & \multicolumn{3}{|c|}{ HTG } \\
\hline & & & & Case MAF & OR $(95 \% C I)$ & $p$-Value & Case MAF & OR $(95 \% C I)$ & $p$-Value \\
\hline ROCK2 & rs2271621 & $\mathrm{G}>\mathrm{T}$ & 0.479 & 0.491 & $1.05(0.81,1.36)$ & 0.71 & 0.468 & $0.96(0.66,1.42)$ & 0.85 \\
\hline ROCK2 & rs202027620 & $G>A$ & 0.005 & 0.000 & & & 0.000 & & \\
\hline ROCK2 & rs2230773 & $\mathrm{C}>\mathrm{T}$ & 0.024 & 0.020 & $0.83(0.34,2.00)$ & 0.67 & 0.039 & $1.59(0.55,4.56)$ & 0.40 \\
\hline ROCK2 & rs3771106 & $G>A$ & 0.433 & 0.453 & $1.09(0.84,1.41)$ & 0.54 & 0.429 & $1.00(0.67,1.47)$ & 0.99 \\
\hline ROCK2 & rs965665 & $G>C$ & 0.002 & 0.011 & $4.94(0.58,41.83)$ & 0.09 & 0.000 & & \\
\hline ROCK2 & rs10178332 & $\mathrm{A}>\mathrm{C}$ & 0.002 & 0.011 & $4.94(0.58,41.83)$ & 0.09 & 0.000 & & \\
\hline ROCK2 & rs6755196 & $G>A$ & 0.002 & 0.011 & $4.92(0.58,41.72)$ & 0.09 & 0.000 & & \\
\hline ROCK2 & rs10929732 & $\mathrm{G}>\mathrm{A}$ & 0.045 & 0.036 & $0.78(0.41,1.49)$ & 0.45 & 0.039 & $0.83(0.33,2.09)$ & 0.69 \\
\hline ROCK1 & rs75122528 & $\mathrm{A}>\mathrm{T}$ & 0.319 & 0.282 & $0.86(0.65,1.13)$ & 0.27 & 0.364 & $1.19(0.82,1.74)$ & 0.36 \\
\hline ROCK1 & rs2847081 & $\mathrm{T}>\mathrm{C}$ & 0.127 & 0.153 & $1.22(0.84,1.77)$ & 0.28 & 0.076 & $0.56(0.28,1.14)$ & 0.09 \\
\hline ROCK1 & rs8089184 & $\mathrm{T}>\mathrm{C}$ & 0.131 & 0.164 & $1.28(0.89,1.84)$ & 0.18 & 0.078 & $0.56(0.29,1.08)$ & 0.07 \\
\hline ROCK1 & rs288980 & $\mathrm{T}>\mathrm{C}$ & 0.474 & 0.475 & $1.00(0.78,1.29)$ & 1.00 & 0.474 & $0.99(0.69,1.42)$ & 0.97 \\
\hline ROCK1 & rs288979 & $A>G$ & 0.129 & 0.157 & $1.26(0.87,1.82)$ & 0.22 & 0.071 & $0.52(0.26,1.03)$ & 0.045 \\
\hline ROCK1 & rs2127958 & $\mathrm{T}>\mathrm{C}$ & 0.445 & 0.441 & $0.99(0.77,1.28)$ & 0.95 & 0.442 & $0.98(0.68,1.40)$ & 0.90 \\
\hline ROCK1 & rs1481280 & $\mathrm{C}>\mathrm{A}$ & 0.312 & 0.280 & $0.88(0.67,1.15)$ & 0.34 & 0.370 & $1.25(0.87,1.82)$ & 0.23 \\
\hline ROCK1 & rs1006881 & $\mathrm{G}>\mathrm{A}$ & 0.129 & 0.159 & $1.28(0.89,1.84)$ & 0.19 & 0.071 & $0.52(0.26,1.03)$ & 0.045 \\
\hline ROCK1 & rs35996865 & $\mathrm{T}>\mathrm{G}$ & 0.129 & 0.156 & $1.26(0.87,1.82)$ & 0.22 & 0.071 & $0.52(0.26,1.03)$ & 0.045 \\
\hline ROCK1 & rs10083915 & $A>G$ & 0.129 & 0.161 & $1.28(0.89,1.83)$ & 0.19 & 0.071 & $0.52(0.26,1.03)$ & 0.045 \\
\hline ROCK1 & rs11873284 & $A>G$ & 0.129 & 0.160 & $1.28(0.89,1.85)$ & 0.18 & 0.071 & $0.52(0.26,1.03)$ & 0.045 \\
\hline ROCK1 & rs1515210 & $C>G$ & 0.181 & 0.185 & $1.05(0.75,1.46)$ & 0.78 & 0.175 & $0.94(0.58,1.52)$ & 0.80 \\
\hline
\end{tabular}

Statistically significant values are shown in bold. NTG: normal-tension glaucoma, HTG: high-tension glaucoma, ROCK: rho-kinase, MAF: minor allele frequency, OR: odds ratio, CI: confidence interval. SNPs rs34945852, rs35768389, rs190769228, and rs1130157 in ROCK2 were monomorphic in cases and controls and were removed from the table.

In the genotype-phenotype correlation analysis, the SNP rs2230773 in ROCK2 was significantly correlated with CCT-adjusted IOP $(p=0.024)$. The average IOP for the major homozygote (CC) was $17.4 \pm 5.8 \mathrm{mmHg}$, and for the heterozygotes (CT), $20.7 \pm 8.6 \mathrm{mmHg}$ (Figure 1). The minor homozygote (TT) was not found. The SNP rs3771106 in ROCK2 was significantly correlated with AXL $(p=0.024)$. The average AXL for the major homozygote (GG) was $24.41 \pm 1.45 \mathrm{~mm}$, for the heterozygotes (GA) $24.81 \pm 1.58 \mathrm{~mm}$, and for the 
minor homozygotes (AA), $24.90 \pm 1.91 \mathrm{~mm}$ (Figure 1). None of the other POAG-related phenotypes showed any significant correlations with the target SNP genotypes.
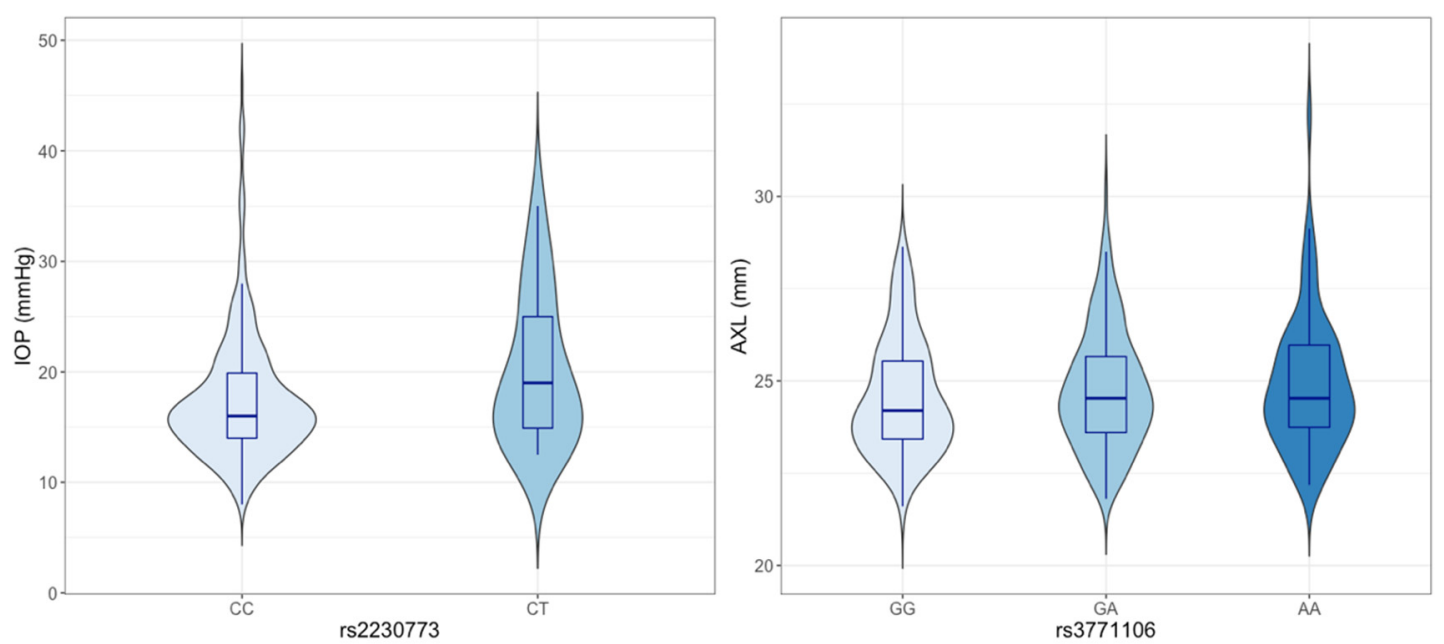

Figure 1. Genotype-phenotype correlations of ROCK-related variants in the entire POAG population. IOP: intraocular pressure, AXL: axial length, ROCK: Rho-associated coiled-coil kinase

In the genotype-phenotype correlation analysis, the SNP rs2230773 in ROCK2 was significantly correlated with CCT-adjusted IOP $(p=0.024)$. The average IOP for the major homozygote (CC) was $17.4 \pm 5.8 \mathrm{~mm} \mathrm{Hg}$, and for the heterozygotes (CT), $20.7 \pm 8.6 \mathrm{~mm}$ $\mathrm{Hg}$. The minor homozygote (TT) was not found. The SNP rs3771106 in ROCK2 was significantly correlated with AXL $(p=0.024)$. The average AXL for the major homozygote (GG) was $24.41 \pm 1.45 \mathrm{~mm}$, for the heterozygotes (GA) $24.81 \pm 1.58 \mathrm{~mm}$, and for the minor homozygotes (AA), $24.90 \pm 1.91 \mathrm{~mm}$.

\section{Discussion}

The present study investigated the effect of ROCK gene polymorphism on the risk of POAG (also NTG and HTG, respectively), and correlated that risk with the relevant clinical factors in a Korean population. Although the data failed to find significant SNPs associated with POAG risk, a subgroup analysis based on baseline IOP revealed that some SNPs in ROCK1 (rs288979, rs1006881, rs35996865, rs10083915, and rs11873284) may be associated with higher-baseline-IOP POAG (i.e., HTG).

ROCK inhibitors are known to reduce IOP by altering TM outflow resistance with the relaxation of smooth muscle cells [6,7]. Besides, ROCK inhibitors are beneficial to glaucoma patients with effects that are independent of IOP: it improves the blood flow to the optic nerve head (via relaxation of vascular endothelial smooth muscle cells) and promotes axonal regeneration [3,9]. To date, two ROCK inhibitors have been approved for clinical use: ripasudil in Japan and netarsudil in the United States. Despite growing clinical interest in ROCK inhibitors as novel therapeutic targets for glaucoma, investigations into ROCK gene polymorphisms for POAG risk have been few. Demiryürek et al. [13] for the first time investigated the effect of $R O C K$ gene polymorphism on POAG risk. They genotyped 8 SNPs in ROCK1 and ROCK2 from 179 POAG patients and 182 healthy controls, but failed to find any significant associations with POAG risk. Our group expanded the number of target SNPs (from 8 to 24) and genotyped for a larger population. We showed that the SNPs rs288979, rs1006881, rs35996865, rs10083915, and rs11873284 in ROCK1 (tagged to each other, $r^{2}=1$ ) were nominally associated with risk of HTG. These SNPs have been reported to be associated with other systemic diseases as well: tetralogy of Fallot for rs288979 [14]; ischemic stroke for rs1006881 and rs10083915 [15], and metabolic syndrome [16], clear cell renal cell carcinoma [17] or systemic sclerosis [18] for rs35996865. As these SNPs were found to be tagged to each other $\left(r^{2}=1\right)$ in the present population, they may be in LD 
with a causative variant rather than being directly causative of POAG risk. Future full sequencing of ROCK1 or ROCK2 will further validate the POAG risk variants.

The absence of significant associations of ROCK gene variants with POAG risk may imply that other effectors in the Rho/ROCK pathway have a role in POAG development. Springelkamp et al. [19] demonstrated that the SNP rs58073046, located within the gene ARHGEF12, was significantly associated with IOP as well as risk of POAG (especially HTG). ARHGEF12 (Rho guanine nucleotide exchange factor (GEF) 12) regulates RhoA GTPases to activate ROCK function, thereby affecting IOP and POAG risk. As clinical evidence for IOP control in POAG patients accumulates, further epigenetics and metabolomics studies promise to uncover complex gene and protein interactions in the Rho/ROCK pathway. Our findings can serve as good reference data for these upcoming studies.

In the present genotype-phenotype correlation analysis, the SNPs rs2230773 and rs3771106 in ROCK2 were significantly correlated with CCT-adjusted IOP and AXL, respectively. The clinical significance of these two SNPs has not yet been established. As the SNP rs2230773 is known to be a synonymous variant, another causal variant near it may have a role in IOP balancing. The SNP rs3771106 is an intronic variant that is known to be tagged with SNPs rs2230774, rs1515219, rs726843, and rs978906. This also suggests that a causal variant may exist in LD with this SNP.

The present study has the following limitations. First, only a small SNP fraction relative to the size of the ROCK gene was selected and genotyped for ROCK polymorphism. This may have affected the negative association with POAG risk. Further inclusion of SNPs or full sequencing analysis could better elucidate the causal variants for POAG. Second, a relatively small number of HTG patients compared to NTG patients may have biased the present association results. Due to the low prevalence of HTG in Korea, the majority of POAG patients had their baseline IOP lower than $21 \mathrm{~mm} \mathrm{Hg}$. Therefore, caution must be taken to interpret current findings from HTG patients, and further study of larger HTG population is needed. Lastly, all of the subjects included in this analysis were Korean, and so our results cannot be generalized to other ethnicities. East Asian countries including Korea have high proportions of NTG among POAG patients, and thus, subjects from these populations may have different glaucoma characteristics from those of subjects representative of other races or regions [20].

\section{Conclusions}

In conclusion, ROCK1-related variants were nominally associated with risk of HTG, but not with NTG, for a Korean population. Also, SNPs rs2230773 and rs3771106 in ROCK2 showed significant correlation with CCT-adjusted IOP and AXL, respectively. The present data supports the role of $R O C K$ in POAG pathogenesis and the relevant key clinical phenotypes, including IOP. This study will serve as an important reference for further investigations of the efficacy of ROCK inhibitors according to ROCK polymorphism.

Supplementary Materials: The following are available online at https://www.mdpi.com/article/10 .3390/jcm10091953/s1, Table S1: title, Video S1: List of Genotyping Primers.

Author Contributions: Conceptualization, Y.-W.K., Y.-J.K., and J.-W.J.; Data curation, E.B., S.W., and S.-C.L.; Funding acquisition, J.-W.J.; Methodology, Y.-W.K., Y.-J.K., and J.-W.J.; Project administration, Y.-W.K. and J.-W.J.; Resources, Y.-W.K., Y.-K.K., K.-H.P., and J.-W.J.; Software, Y.-W.K.; Supervision, Y.-K.K., K.-H.P., and J.-W.J.; Validation, S.W.; Visualization, Y.-W.K.; Writing-original draft, Y.-W.K. and J.-W.J.; Writing-review and editing, Y.-W.K., E.B., S.-C.L., and J.-W.J. All authors have read and agreed to the published version of the manuscript.

Funding: This work was supported by research funds from pH PHARMA Inc., Korea. The funding organization had no role in the design or conduct of this research.

Institutional Review Board Statement: The study was conducted according to the guidelines of the Declaration of Helsinki, and approved by the Institutional Review Board of Seoul National University Hospital (protocol code 1804-063-937, 16 April 2018). 
Informed Consent Statement: Informed consent was obtained from all subjects involved in the study.

Data Availability Statement: The data presented in this study are available on request from the corresponding author. The data are not publicly available due to patient privacy issues.

Acknowledgments: We thank Sanjeev Satyal, Jaesoon Kim, and Hoyoung Huh for their administrative support of our research funding.

Conflicts of Interest: The authors declare no conflict of interest. The sponsors had no role in the design, execution, interpretation, or writing of the study.

\begin{tabular}{|c|c|}
\hline ARHGEF12 & Rho guanine nucleotide exchange factor (GEF) 12 \\
\hline AXL & axial length \\
\hline CCT & central corneal thickness \\
\hline $\mathrm{C} / \mathrm{D}$ & cup-to-disc ratio \\
\hline GCIPL & ganglion cell-inner plexiform layer \\
\hline GLAU-GENDISK & GLAUcoma GENe DIscovery Study in Korea \\
\hline HTG & high-tension glaucoma \\
\hline IOP & intraocular pressure \\
\hline LIM & Lin-11/Isl-1/Mec-3 \\
\hline MD & mean deviation \\
\hline MLC & myosin light chain \\
\hline NTG & normal-tension glaucoma \\
\hline OCT & optical coherence tomography \\
\hline OR & odds ratio \\
\hline POAG & primary open-angle glaucoma \\
\hline RGC & retinal ganglion cells \\
\hline RNFL & retinal nerve fiber layer \\
\hline ROCK & Rho-associated coiled-coil kinase \\
\hline SNP & single-nucleotide polymorphism \\
\hline TM & trabecular meshwork \\
\hline VF & visual field \\
\hline
\end{tabular}

\section{References}

1. Leung, T.; Manser, E.; Tan, L.; Lim, L. A novel serine/threonine kinase binding the Ras-related RhoA GTPase which translocates the kinase to peripheral membranes. J. Biol. Chem. 1995, 270, 29051-29054. [CrossRef] [PubMed]

2. Nakagawa, O.; Fujisawa, K.; Ishizaki, T.; Saito, Y.; Nakao, K.; Narumiya, S. ROCK-I and ROCK-II, two isoforms of Rho-associated coiled-coil forming protein serine/threonine kinase in mice. FEBS Lett. 1996, 392, 189-193. [CrossRef]

3. Inoue, T.; Tanihara, H. Rho-associated kinase inhibitors: A novel glaucoma therapy. Prog. Retin. Eye Res. 2013, 37, 1-12. [CrossRef] [PubMed]

4. Amano, M.; Fukata, Y.; Kaibuchi, K. Regulation and functions of Rho-associated kinase. Exp. Cell Res. 2000, 261, 44-51. [CrossRef] [PubMed]

5. Julian, L.; Olson, M.F. Rho-associated coiled-coil containing kinases (ROCK): Structure, regulation, and functions. Small Gtpases 2014, 5, e29846. [CrossRef] [PubMed]

6. Moura-Coelho, N.; Tavares Ferreira, J.; Bruxelas, C.P.; Dutra-Medeiros, M.; Cunha, J.P.; Pinto Proenca, R. Rho kinase inhibitors-a review on the physiology and clinical use in Ophthalmology. Graefe's Arch. Clin. Exp. Ophthalmol. Albrecht Von Graefes Arch. Fur Klin. Und Exp. Ophthalmol. 2019, 257, 1101-1117. [CrossRef] [PubMed]

7. Tanna, A.P.; Johnson, M. Rho Kinase Inhibitors as a Novel Treatment for Glaucoma and Ocular Hypertension. Ophthalmology 2018, 125, 1741-1756. [CrossRef] [PubMed]

8. Mueller, B.K.; Mack, H.; Teusch, N. Rho kinase, a promising drug target for neurological disorders. Nat. Rev. Drug Discov. 2005, 4, 387-398. [CrossRef] [PubMed]

9. Tokushige, H.; Waki, M.; Takayama, Y.; Tanihara, H. Effects of Y-39983, a selective Rho-associated protein kinase inhibitor, on blood flow in optic nerve head in rabbits and axonal regeneration of retinal ganglion cells in rats. Curr. Eye Res. 2011, 36, 964-970. [CrossRef] [PubMed]

10. Chihara, E.; Dimitrova, G.; Chihara, T. Increase in the OCT angiographic peripapillary vessel density by ROCK inhibitor ripasudil instillation: A comparison with brimonidine. Graefe's Arch. Clin. Exp. Ophthalmol. Albrecht Von Graefes Arch. Fur Klin. Und Exp. Ophthalmol. 2018, 256, 1257-1264. [CrossRef] [PubMed] 
11. Kim, Y.W.; Kim, Y.J.; Cheong, H.S.; Shiga, Y.; Hashimoto, K.; Song, Y.J.; Kim, S.H.; Choi, H.J.; Nishiguchi, K.M.; Kawai, Y.; et al. Exploring the Novel Susceptibility Gene Variants for Primary Open-Angle Glaucoma in East Asian Cohorts: The GLAU-GENDISK Study. Sci. Rep. 2020, 10, 221. [CrossRef] [PubMed]

12. Loirand, G. Rho Kinases in Health and Disease: From Basic Science to Translational Research. Pharm. Rev. 2015, 67, 1074-1095. [CrossRef] [PubMed]

13. Demiryurek, S.; Okumus, S.; Bozgeyik, I.; Oztuzcu, S.; Coskun, E.; Mat, E.; Durucu, E.; Tatar, M.G.; Erbagci, I.; Gurler, B.; et al. Investigation of the Rho-kinase Gene Polymorphism in Primary Open-angle Glaucoma. Ophthalmic Genet. 2016, 37, 9-13. [CrossRef] [PubMed]

14. Palomino Doza, J.; Topf, A.; Bentham, J.; Bhattacharya, S.; Cosgrove, C.; Brook, J.D.; Granados-Riveron, J.; Bu'Lock, F.A.; O'Sullivan, J.; Stuart, A.G.; et al. Low-frequency intermediate penetrance variants in the ROCK1 gene predispose to Tetralogy of Fallot. BMC Genet. 2013, 14, 57. [CrossRef] [PubMed]

15. Zee, R.Y.L.; Wang, Q.M.; Chasman, D.I.; Ridker, P.M.; Liao, J.K. Gene variations of ROCKs and risk of ischaemic stroke: The Women's Genome Health Study. Clin. Sci. 2014, 126, 829-835. [CrossRef] [PubMed]

16. Tabur, S.; Oztuzcu, S.; Oguz, E.; Korkmaz, H.; Eroglu, S.; Ozkaya, M.; Demiryurek, A.T. Association of Rho/Rho-kinase gene polymorphisms and expressions with obesity-related metabolic syndrome. Eur. Rev. Med. Pharmacol. Sci. 2015, 19, 1680-1688. [PubMed]

17. Zhao, R.; Liu, K.; Huang, Z.; Wang, J.; Pan, Y.; Huang, Y.; Deng, X.; Liu, J.; Qin, C.; Cheng, G.; et al. Genetic Variants in Caveolin-1 and RhoA/ROCK1 Are Associated with Clear Cell Renal Cell Carcinoma Risk in a Chinese Population. PLoS ONE 2015, 10, e0128771. [CrossRef] [PubMed]

18. Pehlivan, Y.; Yolbas, S.; Cetin, G.Y.; Alibaz-Oner, F.; Cagatay, Y.; Yilmaz, N.; Oztuzcu, S.; Donmez, S.; Ozgen, M.; Koca, S.S.; et al. Investigation of the association between Rho/Rho-kinase gene polymorphisms and systemic sclerosis. Rheumatol. Int. 2016, 36, 421-427. [CrossRef] [PubMed]

19. Springelkamp, H.; Iglesias, A.I.; Cuellar-Partida, G.; Amin, N.; Burdon, K.P.; van Leeuwen, E.M.; Gharahkhani, P.; Mishra, A.; van der Lee, S.J.; Hewitt, A.W.; et al. ARHGEF12 influences the risk of glaucoma by increasing intraocular pressure. Hum. Mol. Genet. 2015, 24, 2689-2699. [CrossRef] [PubMed]

20. Cho, H.K.; Kee, C. Population-based glaucoma prevalence studies in Asians. Surv. Ophthalmol. 2014, 59, 434-447. [CrossRef] [PubMed] 